\title{
To Studies on the Fauna of the Jewel Beetles (Coleoptera: Buprestidae) of the Nakhchivan Autonomous Republic of Azerbaijan
}

\author{
Ellada Agamelik Huseynova \\ Institute of Zoology, National Academy of Sciences, Baku, Azerbaijan
}

Email address:

ella_line@mail.ru

\section{To cite this article:}

Ellada Agamelik Huseynova. To Studies on the Fauna of the Jewel Beetles (Coleoptera: Buprestidae) of the Nakhchivan Autonomous Republic of Azerbaijan. American Journal of Life Sciences. Vol. 7, No. 3, 2019, pp. 54-60. doi: 10.11648/j.ajls.20190703.11

Received: May 22, 2019; Accepted: June 29, 2019; Published: July 31, 2019

\begin{abstract}
The purpose of this work is to study the current state of the fauna of jewel beetles of the Nakhchivan Autonomous Republic of Azerbaijan. Due to the special geographical position of the Nakhchivan Autonomous Republic, as well as due to the lack of common borders with Azerbaijan, over the past 30 years there have been no special studies on jewel beetles here. Nakhchivan Autonomous Republic is famous for its orchards far beyond the borders of Azerbaijan, and many representatives of the Buprestidae family are serious pests of fruit trees, therefore the study of the species composition is of particular topicality. The peculiarities of the floristic complex of the Nakhchivan Autonomous Republic contributed to the formation of a specific fauna of insects, including jewel beetles. The studies were carried out in the period from 2012 to 2018, beetles collection was carried out from various biotopes during the spring-summer expedition trips that covered all areas of Nakhchivan AR. The paper provides information on the 5 subfamilies and 11 genera. The number of species in subfamilies is distributed as follows: Julodinae - 3, Polycestinae - 4, Chrysochroinae - 8, Buprestinae - 9, Agrilinae - 5. The species Agrilus pratensis RATZEBURG, 1837 is first recorded for the fauna of Azerbaijan.. Our comparative studies show that today the qualitative composition of the Buprestidae family of the Nakhchivan Autonomous Republic is represented by a large number of species than the other two natural regions of Azerbaijan: Azerbaijan's territory of the Greater Caucasus and the Lenkoran natural region. The article presents the taxonomic distribution of species, the places of their gathering, the coordinates of the locality, as well as the worldwide distribution.
\end{abstract}

Keywords: Nakhchivan, Jewel Beetles, Species, Distribution

\section{Introduction}

The first information about the jewel-beetles spread in Azerbaijan is given in the works of Menetrie [1], Babajanidi [2], who recorded 57 species for the Elisavetpol (the former name of Ganja) environs. The works of Richter are of great importance [3]. N.G. Samadov indicates that the fauna of Azerbaijani jewel-beetles includes 171 species, 26 of which are pests of agricultural crops [4]. M.G. Volkovich recorded 151 species of jewel-beetles from Azerbaijan [5]. S. Billy works also include species from Azerbaijan [6]. The natural conditions of Azerbaijan are heterogeneous and very interesting in faunistic point of view. The flora and fauna of some areas include relict species. In modern conditions of strong anthropogenic impact, the processes of transformation and depletion of natural cenoses take place. In this regard, the study of the faunistic composition and ecological features of the jewel beetles of various natural areas of the country is very relevant. In neighboring countries of Azerbaijan, the study of this group of beetles is also of great interest among researchers in Russia [7], Turkey [8, 9], Iran [10], Armenia [11], Turkmenistan [12], Kazakhstan [13].

Nakhchivan AR occupies the south-western part of the Caucasus. Almost $75 \%$ of the territory lies at an altitude of more than $1000 \mathrm{~m}$. On the territory of Nakhchivan AR there is rare flora and fauna of Azerbaijan. The south and southwestern parts of the territory along the Araks River are a plain of $600-1000 \mathrm{~m}$ in height. Although the territory of the Autonomous Republic is $6.2 \%$ of the entire territory of the Republic of Azerbaijan, according to species diversity its 
flora and fauna represents respectively about $60 \%$ and $56 \%$ flora and fauna of the country. In the territory of Nakhchivan $\mathrm{AR}$, the lower vegetative cover and its types are divided into: deserts and semi-deserts; phryganoid and steppe vegetation; mountain meadows and steppes; high grass, subalpine and alpine meadows [14]. A list of 29 species of beetles belonging to 11 genera and 5 subfamilies is presented below. The species Agrilus pratensis RATZEBURG, 1837 is first recorded for the fauna of Azerbaijan.

\section{Material and Methods}

Material. The species collected by senior author, as well as by colleagues used as a material for paper. The regular collections were conducted in the spring and summer seasons during 2012-2018 by standard entomological methods [15]. Species determination was carried out by keys [16].

Distibution. The separate list containing the names and coordinates of collection sites in Nakhchivan AR of Azerbaijan is given.

Taxonomic and nomenclatural acts. The taxonomic position, status, geographical distribution and nomenclature of the species corresponds to the modern system [5], the names are given in alphabetical order. Localities and dates of collection are listed in chronological order.

Mapping. Map was generated by exporting distributional data from the relational database. The collections of the beetles were conducted by generally-used entomological sampling methods, during the expedition trips to the regions of Nakhchivan AR (Babek, Julfa, Shahbuz, Sharur, Ordubad, Nakhchivan) covering spring-summer period from 2012 to 2018.

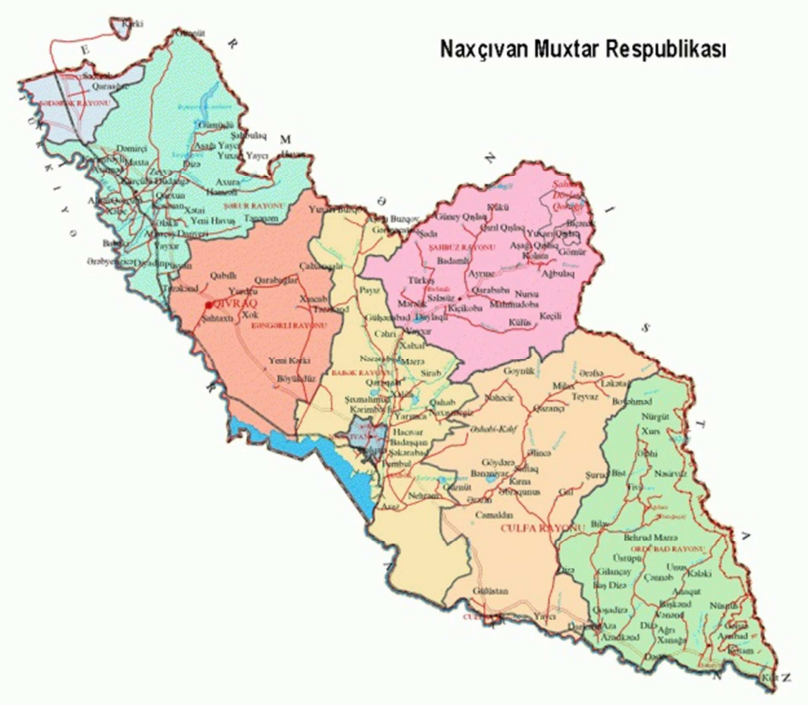

Figure 1. Map of the Nakhchivan Autonomous Republic of Azerbaijan.

\section{Results}

In a previously published paper on the fauna of the jewel beetlesof Nakhchivan AR, 18 species were presented [17].
Over the past period, another 11 were added to this number. Below is a list of 29 species of jewel beetles collected in the Nakhchivan Autonomous Republic in accordance with their taxonomic position, an indication of the collection sites and localities. Pictures of some of them are also presented.

Family BUPRESTIDAE LEACH, 1815

Subfamily Julodinae LACORDAIRE, 1857

Genus Julodella SEMENOV, 1893

Julodella dilaticollis SEMENOV, 1893

Material: Julfa, Erefse, 30.06.2018, N39 ${ }^{0}$ 17 06.4" E045 46'59,7", 1591 m, I.KERIMOVA.6 sp.: Sharur, Tezekend, 29.06.2018, N39 24 59, $8^{\prime \prime}$ E045 01'53,3", 777 m, I.KERIMOVA. $1 \mathrm{sp}$.

Distribution: Europe: Azerbaijan, Georgia. Asia: Iran, Turkey.

Genus Julodis ESCHSCHOLTZ, 1829

Julodis andreae andreae (OLIVIER, 1790)

Material: Sirab, 23.06.12,N39 14 826.4" E045 ${ }^{\circ} 26^{\prime} 388^{\prime \prime}, \mathrm{H}$ - 924 m, E.HuSEYNOVA. 4 sp.; Ordubad, Aghdara, 22.07.17,

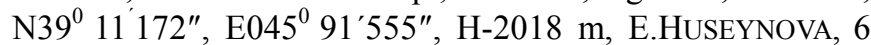
sp; Shakhbuz, 02.07.18, N39 $52^{\prime} 840^{\prime \prime}$ E045 $77^{\prime} 49,0^{\prime \prime}$, H1919 m, I.KERIMOVA. $1 \mathrm{sp.}$

Distribution: Europe: Azerbaijan, Armenia. Asia: Iran, Iraq, Turkey. Syria

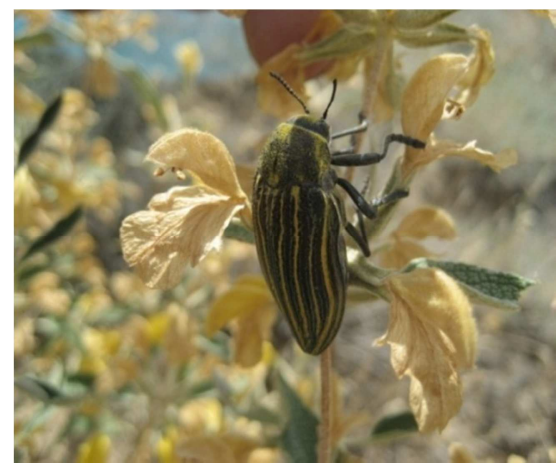

Figure 2. Julodis andreae andreae (HUSEYNOVA E.).

Julodis variolaris variolaris PALLAS, 1771

Material: Ordubad, Aghdara, 22.07.17, N39 ${ }^{0} 11$ 172" E045 91'555", H-2018 m. E.HuSEYNOVA, 1 sp.

Distribution: Europe: Azerbaijan, Kazakhstan, Russia (South European Territory). Asia: Afghanistan, China (Gansu), Iran, Kyrgyzstan, Kazakhstan, Turkmenistan, Turkey, Uzbekistan, China (Xinjiang).

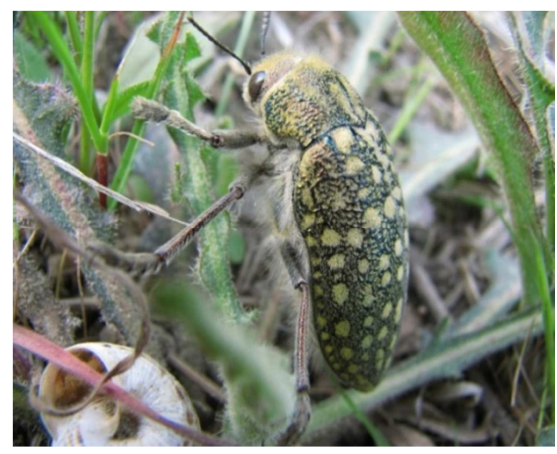

Figure 3. Julodis variolaris variolaris (HUSEYNOVA E.). 
Subfamily Polycestinae LACORDAIRE, 1857

Tribe Acmaeoderini KERREMANS, 1893

Genus Acmaeoderella CoBOS, 1955

Subgenus Carininota VOLKOVITSH, 1979

Acmaeoderella flavofasciata albifrons (ABEILLE de PERRIN, 1891)

Material: Ordubad, 25.06.12, N39 $9^{0}$ 07 594" E $045^{\circ}$ 26'388", H - 1199m, E.HuSEYNOVA, 1 sp; Shakhbuz, 02.07.18, H-1919m: N-39.52 $840^{\circ} \quad$ E-045.77 $490^{\prime \prime}$. I.KerIMOVA1 sp.; Shakhbuz, Kolani, 02.07.2018. N39 ${ }^{0}$ $27^{\prime} 03.33^{\prime \prime}$ E $045^{\circ}$ 40’34.89", H - 1393m. I.KeRIMOVA 3 sp.

Distribution: Europe: Azerbaijan, Armenia. Asia: Turkey. Subgenus Euacmaeoderella VoLKOVITSH, 1979

Acmaeoderella gibbulosa MÉNÉTRIÉS, 1832

Material: Ordubad, Aghdara, 22.07.17. N39 11 172"

E045 91'555", H-2018 m, E.HUSEYNOVA, 2 sp.

Distribution: Europe: Azerbaijan, Armenia. Bulgaria, Croatia, Macedonia. Asia: Cyprus, Iran, Iraq, Israel, Jordan, Kazakhstan, Lebanon, Syria, Turkmenistan, Turkey.

Acmaeoderella vetusta (MÉNÉTRIÉS, 1832)

Material: Ordubad, Behrud, 27.06.12, N39 $0432.16^{\prime \prime} \mathrm{E}$ 045 51'48.87", H - 1348 m, E.HuSEYNOVA, 8 sp.; Ordubad, Aghdara, 22.07.17, N39 $11^{\prime} 172^{\prime \prime}$ E045 $91^{\prime} 555^{\prime \prime}$, H-2018 m, E.HuSEYNOVA, 2 sp.; Ordubad, Aghdara, 06.07.18, $10 \mathrm{sp}$; Kengerli, 03.07.18, N39 ${ }^{0} 23.57 .94^{\prime \prime}$ E $045^{0} 10^{\prime} 59.73^{\prime \prime}$, I.KERIMOVA, $\mathrm{H}-1074 \mathrm{~m} .1 \mathrm{sp}$.

Distribution: Europe: Azerbaijan, Armenia. Bulgaria, Croatia. Asia: Cyprus, Iran, Iraq, Israel, Jordan, Lebanon, Syria, Turkmenistan, Turkey.

Acmaeoderella villosula STEVEN, 1830

Material: Ordubad, Aghdara, 06.07.18. N39 11 172" E045 91'555", H-2018 m, I.KERIMOVA, 2 sp.

Distribution: Europe: Azerbaijan, Armenia, Georgia, Croatia, Macedonia. Asia: Afghanistan, Cyprus, Iran, Iraq, Israel, Jordan, Lebanon, Syria, Tajikistan, Turkmenistan, Turkey.

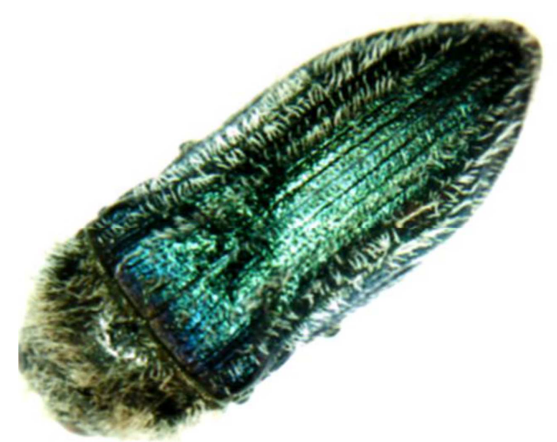

Figure 4. (USB) Acmaeoderella villosula (HUSEYNOVA E.).

SubfamilyChrysochroinaeLAPORTE, 1835

Tribe Dicercini GISTEL, 1848

Genus Capnodis EsCHSCHOLTZ, 1829

Capnodis miliaris miliaris (KLUG, 1829)

Material: Ordubad, Bilav, 26.06.12. N39 07 $0794^{\prime \prime}$ E045

26'388', H - 1199 m, E.HuSEYNOVA. 1 sp.

Distribution: Europe: Azerbaijan, Armenia, Georgia, Italy,
Russia (South European Territory). Asia: Cyprus, Iran, Iraq, Israel, Jordan, Kyrgyzstan, Kazakhstan, Lebanon, Syria, Turkmenistan, Turkey, Uzbekistan.

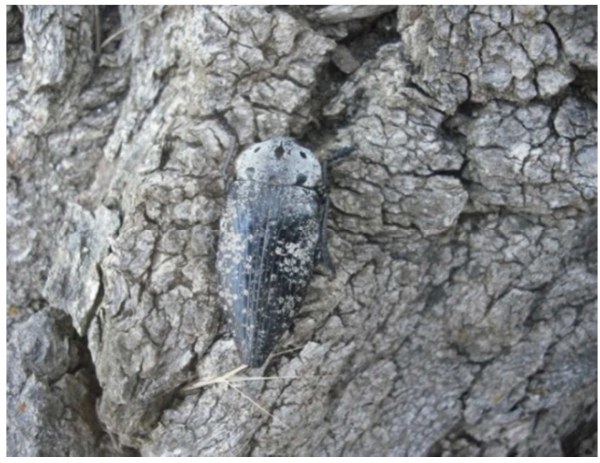

Figure 5. Capnodis miliaris miliaris (HUSEYNOVA E.).

Genus Perotis DEJEAN, 1833

Perotis cuprata (KLUG, 1829)

Material: Sirab, 07.06.17, N39 $144^{\prime} 826.4^{\prime \prime}$ E045 $26^{\prime} 388^{\prime \prime}$, H - 924 m, E.HuSEYNOVA. 1 sp.

Distribution: Europe: Azerbaijan, Armenia. Asia: Iran, Iraq, Jordan, Lebanon, Syria, Turkey.

Perotis lugubris longicollis KRAATZ, 1880

Material: Ordubad, Aghdara, 28.06.12,N39 11'172" E045

91'555"H-2018 m, E.HUSEYNOVA. 3 sp.

Distribution: Europe: Azerbaijan, Armenia, Georgia. Asia: Iran, Iraq, Turkmenistan, Turkey.

Tribe Sphenopterini LACORDAIRE, 1857

Genus Sphenoptera DEJEAN, 1833

Subgenus Chrysoblemma JAKOVLEV, 1889

Sphenoptera orichalcea (PALLAS, 1781)

Material: Julfa, 01.07.2018. N-38 56 52.29", E-045 ${ }^{0} 37^{\prime}$ 11.22", H 804, I.KeRIMOVA, 1 sp.

Distribution: Europe: Azerbaijan, Armenia, Kazakhstan, Russia (Central and South European Territory). Asia: Kyrgyzstan, Kazakhstan, Mongolia, China (Northern Northwest Territory), Tajikistan, Turkmenistan, Uzbekistan. Russia (West Siberia).

Sphenopterascovitzii scovitziiFALDERMANN, 1835

Material: Ordubad, 25.06.12. N-39 $07^{\prime} 594^{\prime \prime} \mathrm{E}-045^{0} 26^{\prime}$

388", H - 1199m, E.HUSEYNOVA.1 sp.

Distribution: Europe: Azerbaijan, Armenia, Russia (South European Territory). Asia: Afghanistan, Iran, Iraq, Israel, Kazakhstan, Tajikistan, Turkmenistan, Turkey, Uzbekistan.

Subgenus Deudora JAKOVLEV, 1899

Sphenoptera sculpticollis HEYDEN, 1886

Material: Chalkhan Gala, 02.05.12. N-39 $19256^{\prime \prime}$. E $045^{0}$ 50005". H-1900, E.HuSEYNOVA 1 sp.

Distribution: Europe: Azerbaijan, Armenia, Georgia. Asia: Cyprus, Iran, Iraq, Israel, Lebanon, Syria, Turkey.

Sphenoptera simplexJAKOVLEV, 1893

Material: Ordubad, Agdara, 28.07.2017. N-390 11 172", E045 91'555", -2018 m,E.HuSEYNOVA. 1 sp.

Distribution: Europe: Azerbaijan, Armenia Greece (Thracia, Aegean Islands) Asia: Iran, Turkey.

Subgenus Sphenoptera DEJEAN, 1833 


\section{Sphenoptera tragacanthae KLUG, 1829}

Material: Ordubad, Agdara, 22.07.2017, N-390 11'172", E$045^{0} 91^{\prime} 555^{\prime \prime}, \mathrm{H}-2018 \mathrm{~m}$, E.HUSEYNOVA. 11sp.; Shakhbuz, 02.07.2018, N-39 ${ }^{0} .52^{\prime} 840^{\prime \prime}$ E- $045^{0} \quad 77^{\prime} 490^{\prime \prime}$ H-1919m, I.KERIMOVA. $1 \mathrm{sp}$.

Distribution: Europe: Azerbaijan, Armenia, Georgia. Greece, Ukraine (Krym) Asia: Afghanistan, Iran, Iraq, Kyrgyzstan, Lebanon, Pakistan, Syria, Turkmenistan, Turkey.

Subfamily Buprestinae LEACH, 1815

Tribe Anthaxiini Gory \&LAPORTE, 1837

Genus Anthaxia ESCHSCHOLTZ, 1829

Subgenus Anthaxia ESCHSCHOLTZ, 1829

Anthaxia bicolor bicolor FALDERMANN, 1835

Material: Shakhbuz, 3.05.12,N-39 ${ }^{0} .52^{\prime} 840^{\prime \prime}$ E- $045^{0}$ $77^{\prime} 490 "$, H-2018 m, E.HuSEYNOVA.12 sp.

Distribution: Europe: Azerbaijan, Albania, Armenia, Bulgaria, Croatia, Georgia, Greece, Macedonia, Romania, Russia (South European Territory), Turkey, Ukraine (Krym). Asia: Iran, Iraq, Israel, Jordan, Lebanon, Syria, Turkmenistan, Turkey.

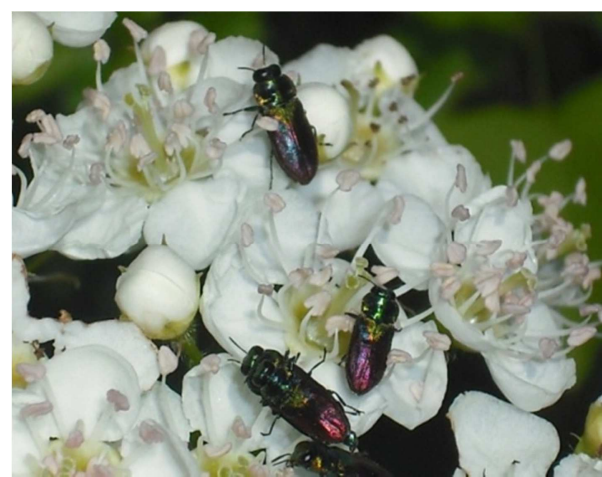

Figure 6. Anthaxia bicolor bicolor (HUSEYNOVA E.).

Anthaxia ephippiata L. REDTENBACHER, 1850

Material: Shakhbuz, 3.05.12,N-39 ${ }^{0} .52^{\prime} 840^{\prime \prime}$ E- $045^{0}$ $77^{\prime} 490 "$ H-2018 m, E.HuSEYNOVA. $1 \mathrm{sp.}$

Distribution: Europe: Azerbaijan, Armenia, Georgia. Asia: Afghanistan, Iran, Iraq, Syria, Turkmenistan, Turkey.

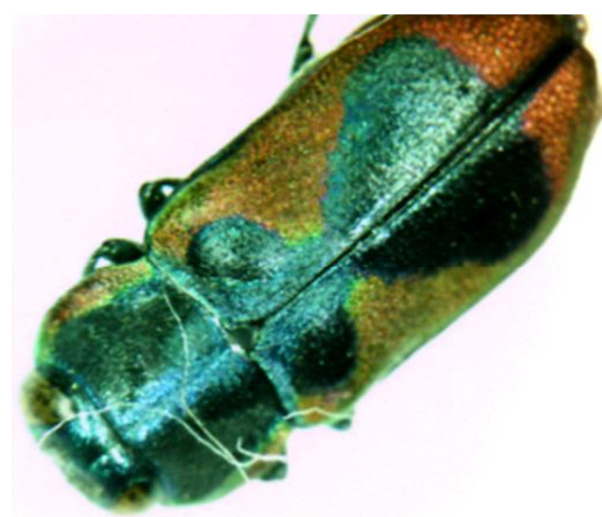

Figure 7. (USB) Anthaxia ephippiata (HUSEYNOVA E.).

Anthaxia muliebris OBENBERGER, 1918

Material: Ordubad, Behrud, 27.06.12, N-39 04 32.16", E045 51'48.87", H - 1348 m, E.HuSEYNOVA.1sp. Ordubad,
Bilav, 26.06.12, N-390 07 594", E-045 26'388", H-1199m. E.HUSEYNOVA. $3 \mathrm{sp.}$

Distribution: Europe: Azerbaijan, Armenia, Turkey. Asia: Iran, Israel, Syria, Turkmenistan, Turkey.

SubgenusCratomerusSOLIER, 1833

Cratomerus diadema shelkovnikoviOBENBERGER, 1940

Material: Ordubad, Behrud. 27.06.12. N-39 04 32.16", E045 51'48.87", H - 1348 m, E.HuSEYNOVA. 2 sp.

Distribution: Europe: Azerbaijan, Armenia, Georgia. Asia: Iran

Cratomerus hungaricus sitta (KÜSTER, 1852)

Material: Shakhbuz, 23.06.12. N-39 ${ }^{0} .52^{\prime} 840^{\prime \prime}$, E- $045^{0}$ $77^{\prime} 490^{\prime \prime}$, H-1919m, E.HuSEYNOva.4 sp.; Ordubad Bilav, 26.06.12. N-39 $07^{\prime} 594^{\prime \prime}$ E- $045^{0}$ 26'388", H - 1199m. E.HUSEYNOVA. $7 \mathrm{sp.}$

Distribution: Europe: Azerbaijan, Armenia Georgia, Russia (South European Territory), Ukraine. Asia: Iran, Turkmenistan, Turkey.

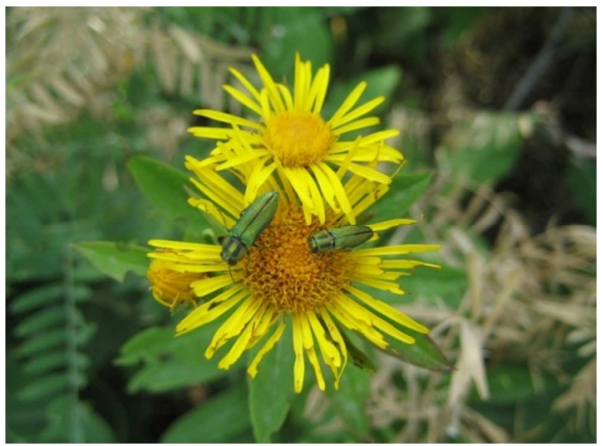

Figure 8. Cratomerus hungaricus sitta (KERIMOVA I.).

Cratomerus mirabilis (ZHIKHAREV, 1918)

Material: Shakhbuz, 23.06.12. N-39 ${ }^{0} .52^{\prime} 840^{\prime \prime}$ E-045 77 490" H-1919 m, E.HuSEYNOVA. 2 sp.

Distribution: Europe: Azerbaijan, Armenia. Asia: Turkey.

Cratomerus sponsa (KIESENWETTER, 1857)

Material: Ordubad, Bilav, 26.06.12,N-39 07 594", E-045 26'388", H - 1199m. E.HuSEYNOVA.3 sp.

Distribution: Europe: Azerbaijan, Armenia, Bulgaria, Georgia, Greece, Macedonia, Romania, Turkey. Asia: Iran, Israel, Jordan, Lebanon, Syria, Turkmenistan, Turkey.

Subgenus Haplanthaxia REITTER, 1911

Anthaxia cichorii (OLIVIER, 1790)

Material: Shakhbuz, 3.05.12. N-39 ${ }^{0} .52^{\prime} 840^{\prime \prime}$ E-045 77 490" H-1919m, E.HuSEYNOVA. 3 sp.; Sirab, 23.06.12, N39 14 826.4" E045 26'388", H - 924 m, E.HUSEYNOVA. 4 sp.; Ordubad, 25.06.12. N-39 $07^{\prime} 594^{\prime \prime} \mathrm{E}-045^{0} 26^{\prime} 388^{\prime \prime}, \mathrm{H}-$ 1199 m, E.HuSEYNOVA. 4 sp.; Agdara, 05.07.2018, N39 $11^{\prime} 172^{\prime \prime}$ E045 91'555"H-2018 m, I.KeRIMOVA, 2 sp.

Distribution: Europe: Azerbaijan, Albania, Armenia, Austria, Belgium, Bosnia Herzegovina, Bulgaria, Croatia, Russia (Central and South European Territory), Czech Republic, France, Germany, Georgia, Greece, Hungary, Italy, Macedonia, Moldova, Montenegro, Poland, Romania, Serbia, Slovakia, Slovenia, Spain, Switzerland, Turkey, Ukraine. Asia: Iran, Iraq, Israel, Jordan, Lebanon, Syria, Turkmenistan, Turkey. 


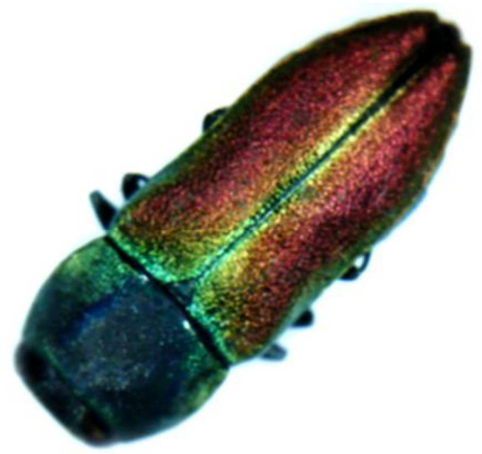

Figure 9. (USB) Anthaxia cichorii (HUSEYNOVA E.).

Tribe Melanophilini BEDEL, 1921

Genus Trachypteris KIRBY, 1837

Trachypteris picta decostigma FABRICIUS, 1787

Material: Shakhbuz, 27.07.16. N-39 $9^{0} 52^{\prime} 840^{\prime \prime}$ E- $045^{0}$ 77 490"'. H-1919m, I.KERIMOVA, 8 sp.

Distribution: Europe: Azerbaijan, Albania, Armenia, Austria, Bosnia Herzegovina, Belarus, Croatia, Czech Republic, France, Georgia, Greece, Hungary, Italy, Macedonia, Moldova, Montenegro, Portugal, Romania, Serbia, Slovakia, Switzerland, Turkey, Ukraine. North Africa: Algeria, Morocco, Tunisia. Asia: Cyprus, Iran, Iraq, Israel, Syria, Turkey.

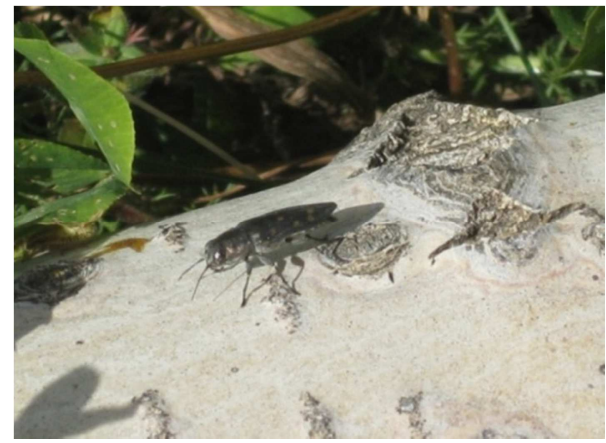

Figure 10. Trachypteris picta decostigma (KERIMOVA I.).

Subfamily Agrilinae LAPORTE, 1835

Tribe Agrilini LAPORTE, 1835

Subtribe Agrilina LAPORTE, 1835

Genus Agrilus CURTIS, 1825

Agrilus pratensis RATZEBURG, 1837

Material: Shakhbuz, 23.06.12, 10.06.2018, N-39 ${ }^{0} 52^{\prime} 840^{\prime \prime}$

E-045 77 490",H-1393 m, E.HuSEYNOVA, 2 sp,

Distribution: Europe: Albania, Armenia, Austria, Belgium, Bosnia Herzegovina, Bulgaria, Belarus, Croatia, Czech Republic, Denmark Finland France, Great Britain, Germany, Georgia, Greece, Hungary, Italy, Kazakhstan, Latvia Liechtenstein, Lithuania, Luxembourg, Macedonia, Moldova, Montenegro, Netherlands, Norway, Poland, Romania, Serbia, Slovakia, Slovenia, Spain, Russia (North Central South European Territory), Sweden, Switzerland, Turkey, Ukraine. Asia: Russia (West, East and Far East Siberia), Iran, Kazakhstan, Mongolia, China, Turkey. Agrilus viridis LINNAEUS, 1758
Material: Shakhbuz, 23.06.12,N-39 ${ }^{0} 52^{\prime} 840^{\prime \prime} \quad$ E- $045^{0}$ 77 490", H-1393 m, E.HuSEYNOVA. 11 sp.

Distribution: Europe: Azerbaijan, Albania, Armenia, Austria, Belgium, Bosnia Herzegovina, Bulgaria, Belarus, Croatia, Czech Republic, Denmark, Finland, France, Great Britain, Germany, Georgia, Greece, Hungary, Italy, Kazakhstan, Latvia Liechtenstein, Lithuania, Luxembourg, Macedonia, Montenegro, Netherlands, Norway, Poland, Portugal, Romania, Serbia, Slovakia, Spain, Russia (North Central South European Territory), Sweden, Switzerland, Turkey, Ukraine. North Africa: Algeria, Morocco, Madeira Archipelago, Tunisia. Asia: China, Russia (West, East and Far East Siberia), Iran, Japan Kyrgyzstan, Kazakhstan, Mongolia, Turkey, Uzbekistan.

Tribe Coraebini BEDEL, 1921

Subtribe Coraebina BEDEL, 1921

Genus Coraebus GORY\&LAPORTE, 1839

Coraebus elatus (FABRICIUS, 1787)

Material: Shakhbuz, 23.06.12.N-39 ${ }^{0} 52^{\prime} 840^{\prime \prime}$ E-045 77 490", H-1393 m, E.HuSEYNOVA.7 sp.

Distribution: Europe: Azerbaijan, Albania, Armenia, Austria, Belgium, Bosnia Herzegovina, Bulgaria, Belarus, Croatia, Czech Republic, France, Germany, Georgia, Greece, Hungary, Italy, Macedonia, Montenegro, Poland, Portugal, Romania, Russia (South, Central and North European Territory), Serbia, Slovakia, Slovenia, Spain, Switzerland, Turkey, Ukraine. North Africa: Algeria, Egypt. Asia: Iran, Iraq, Israel, Kazakhstan, Syria, Turkmenistan, Turkey, Russia (West Siberia).

Coraebus rubi (LINNAEUS, 1767)

Material: Ordubad, Agdara, 05.07.2018. N-39 11 172" E045 91'555"H-2018 m, I.KERIMOVA. 4 sp.

Distribution: Europe: Azerbaijan, Albania, Armenia, Austria, Bosnia Herzegovina, Bulgaria, Croatia, Czech Republic, France, Germany, Georgia, Greece, Hungary, Italy, Luxembourg, Macedonia, Montenegro, Poland, Portugal, Romania, Serbia, Slovakia, Slovenia, Spain, Russia (South European Territory), Switzerland, Turkey, Ukraine. North Africa: Algeria, Morocco. Asia: Cyprus, Iran, Iraq, Israel, Lebanon, Syria, Turkey.

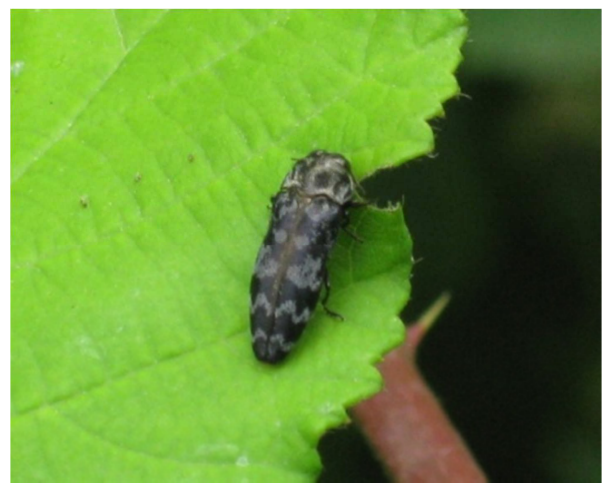

Figure 11. Coraebus rubi (KERIMOVA I.)

Subtribe Meliboeina MAJER, 2000

Genus Meliboeus DeYRoLLE, 1864 


\section{Meliboeus robustus (KÜSTER, 1852)}

Material: Ordubad, Agdara, 28.06.12, N-39 $19^{\prime} 172^{\prime \prime}$, E045 91'555", H-2018 m, E.HUSEYNOVA. 5 sp.

Distribution: Europe: Azerbaijan, Armenia, Georgia, Russia (South European Territory). Asia: Iran, Iraq, Turkmenistan, Turkey.

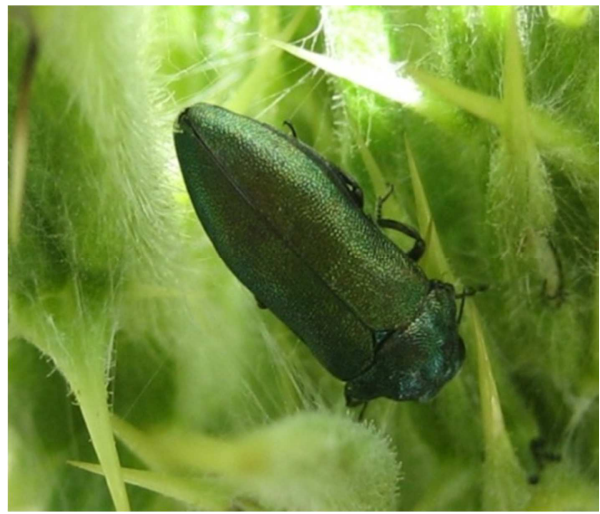

Figure 12. Meliboeus robustus (HUSEYNOVA E.).

As the diagram 1 shows, the largest number of species in the studied region belongs to the subfamily Buprestinae, and the smallest one to Julodinae.

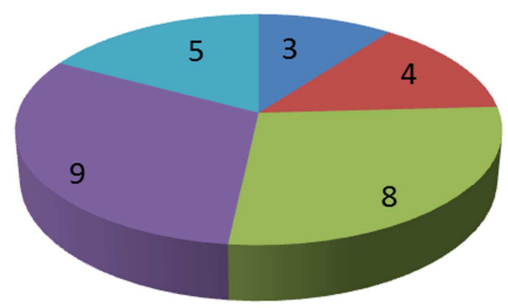

\section{- Julodinae \\ Polycestinae \\ Chrysochroinae \\ Buprestinae \\ agrilinae}

Figure 13. Numerical ratio of species by subfamily.

The compare of the number of species of jewel beetles in the Nakhchivan Autonomous Republic with other regions of Azerbaijan revealed, that it constitutes the majority making $\% 41$.

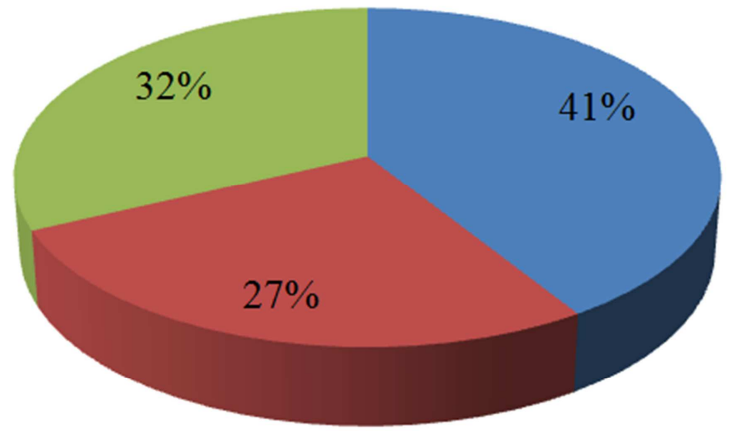

\section{Naxcivan $\square$ Lankaran $\square$ Greeate Caucasus}

Figure 14. Percentage ratio of species of jewel beetles in 3 natural areas of Azerbaijan.

\section{Conclusion}

This article is part of the work devoted to the study of the current state of the beetles of the Buprestidae family of Azerbaijan. The species composition of the jewel beetles of the Nakhchivan Autonomous Republic differs from other natural areas of Azerbaijan in particular diversity, 29 species presented here belong to 5 subfamilies (data are presented in the diagrams 13, 14). One of them Agrilus pratensis RATZEBURG, 1837 is new to Azerbaijan. Species collection covered all areas of the autonomous republic, which are presented on the map. The geographical coordinates of the sites of the finds and the distribution of beetles in the world according to literary sources are indicated. The work is illustrated with 11 original pictures of beetles.

\section{References}

[1] Menetries E. 1832.Catalogue raisonne des objets de Zoogie requeillis dans un voyage en Caucase et jusquaux frontiers actuelles de la Perse. St.Peetersbourg.

[2] Babadjanidi I. D. 1917. Perechen zlatok, naydennix v okr. Qoroda Elizavetpol. Izv/Cavcaz.muzeya. X, 4.321-324.

[3] Rixter A. A. 1944. Obzor zlatok Evropeyskoy chasti SSSR (Coleoptera, Buprestidae). «Izv.AN Arm.SSR» estestv.nauki. 4.

[4] Samadov N. Q. 1963.Fauna I biologiya jukov vredyashix selskoxozyaystvennim kulturam v Azerbaydjane. Baku s. 1628 .

[5] KubáŇ, V., Jendek, E., Kalashian, M. Yu.\& Volkovitsh, M.G. 2016. Superfamily BUPRESTOIDEA Leach, 1815. (pp. 19-32 [New Acts], 432-574 [Catalogue]). I. Löbl and D. Löbl (eds.). Catalogue of Palaearctic Coleoptera (Revised and Updated Edition).Volume 3. Scarabaeoidea, Scirtoidea, Dascilloidea, Buprestoidea and Byrrhoidea. BRILL. LeidenBoston983 pp.

[6] BíLÝ S. 2002: Summary of the binomy of the Buprestid beetles of Central Europe (Coleoptera: Buprestidae). Acta Entomologica Musei Nationalis Pragae, Supplementum 10: 1-104.

[7] Volkovitsh, M. G. 1986: Obzor zlatok triby Acmaeoderini (Coleoptera, Buprestidae) fauny SSSR i sopredel'nykh stran, p. 16-43 (in Russian with English summary), In: Kirejtshuk, A. G. (ed): Morfologiya, sistematika i faunistika maloizuchennykh grupp nasekomykh. Trudy Zoologicheskogo instituta AN SSSR, Leningrad, Tom 140: 100 pp.

[8] BOLU, H.; OZGEN, I. 2011. On the Buprestidae (Coleoptera) species of almond orchards in the Southern and Eastern Anatolia in Turkey. Munis Entomology and Zoology 6 (2): 970-975.

[9] HACI HÜSEYIN CEBEC. 2018. First record of the jewel beetle Anthaxia midasmidas (Coleoptera, Buprestidae) in Anatolia (Turkey, Asia) Revista Colombiana de Entomología 44 (1): 135-137 (Enero - Junio 2018).

[10] HASSAN GHAHARI, MARK G. VOLKOVITSH, CHARLES L. BELLAMY Vol. 3984. N 11-141 2015 An annotated catalogue of the Buprestidae of Iran (Coleoptera: Buprestoidea). 
[11] Kalashan M. J. 1985. Redkie, maloizvestnie b novie dlya Armenii vidi jukov-zlatok (Coleoptera, Buprestidae). Biol.j. Armenii. 38 (8), 1985: 718-720.

[12] Volkovitsh, M. G. \& Alexeev, A. V. 1994: Buprestid beetles (Coleoptera: Buprestidae) from Kopetdagh and the adjacent regions of Southern Turkmenistan. p. 419-449, In: Fet, V. \& Atamuradov, K. I. (eds): Biogeography and Ecology of Turkmenistan. Dordrecht, Kluwer Academic Publishers.

[13] Tleppaeva A. M. 2014. Jevel-beetls (Coleoptera: Buprestidae) of „Altyn Emel“ State National Natural Park. (Kazachstan). Caucasian Entomological Bulleten. Vol. 10 (1): 77-83.

[14] IBRAgimova A., Nabiyeva F. Flora visokoqoriy Nahchivanskiy AR. Lambert Academic Publishing. 2013. 88 p.
[15] Fasulaty K. K. Polevoe izuchenie nazemnix bespozvonochnix. ,Visshaya shkola“. 1971. Moskva.

[16] Rixter A. A., Alekseev A. V. Sem. Buprestidae - Zlatki. V: Bey-Bienko G.J. (Red.). Opredelitel nasekomix Evropeyskoy chasti SSSR. 2 t. Jestkokrilie i veerokrilie. M.-L., 1965: 283$303 \mathrm{pp}$.

[17] HuSEYNOva E. Juki-zlatki (Coleoptera: Buprestidae) Nahchivanskiy AR Azerbaydjana «Bioraznoobrazie I ratsionalnoe ispolzovanie prirodnix resursov». Materiali IIV vserossiiskoi naucho-prakticheskoi konf. s mejdunarodnim uchastiem Makhachkala. 2014. 90-94 pp. 